\title{
FREE AND LOCALLY FREE ARRANGEMENTS WITH A GIVEN INTERSECTION LATTICE
}

\author{
SERGEY YUZVINSKY
}

(Communicated by Jeffrey N. Kalm)

\begin{abstract}
In previous papers the author characterized free arrangements of hyperplanes by the vanishing of cohomology of the intersection lattice with coefficients in a certain sheaf of graded modules over a polynomial ring. The main result of this paper is that for a locally free arrangement the degrees of nonzero homogeneous components of the cohomology modules are bounded by a number depending only on the intersection lattice. In particular, the Hilbert coefficients of the module of derivations of a locally free arrangement are combinatorial invariants. Another result of the paper asserts that the set of free arrangements is Zariski open in the set of all arrangements with a given intersection lattice.
\end{abstract}

\section{INTRODUCTION}

This paper can be viewed as a continuation of [Y1, Y2] where a certain sheaf cohomology was applied to the theory of arrangements.

Let $V$ be an $l$-dimensional $(l \geq 2)$ vector space over an arbitrary field $K$ and $\mathscr{A}$ an arrangement of $n \quad(n \geq 2)$ hyperplanes in $V$. For each $H \in \mathscr{A}$ we fix a linear functional $\alpha_{H} \in V^{*}$ such that $\operatorname{Ker} \alpha_{H}=H$ and denote the set of these functionals also by $\mathscr{A}$. Let $S=\bigoplus_{d \geq 0} S_{d}$ be the graded symmetric algebra of $V^{*}$ and $\operatorname{Der} S=\operatorname{Der}_{K}(S, S)$ the graded $S$-module of derivations of $S$. The following graded submodule of $\operatorname{Der} S$ was introduced by Terao [T1, T3] and studied in many papers on arrangements:

$$
D=D(\mathscr{A})=\left\{\theta \in \operatorname{Der}(S) \mid \theta\left(\alpha_{H}\right) \in S \alpha_{H}, H \in \mathscr{A}\right\} .
$$

If the module $D$ is free then the arrangement $\mathscr{A}$ is called free. In any case for any minimal system of homogeneous generators of $D$ the degrees of the generators are uniquely defined by $\mathscr{A}$. They are called the structure constants of $\mathscr{A}$ and also the exponents of $\mathscr{A}$ if $\mathscr{A}$ is free.

Let $L$ be the lattice of all intersections of hyperplanes from $\mathscr{A}$ ordered by inclusion. We denote by $U$ the minimal element of $L \quad\left(U=\bigcap_{H \in \mathscr{A}} H\right)$ and put $L_{0}=L \backslash\{U\}$. If $U=0$, the arrangement is called essential. Every $X \in L_{0}$ defines the subarrangement $\mathscr{A}_{X}=\{H \in \mathscr{A} \mid H \supset X\}$ of $\mathscr{A}$ and, hence, an $S$ module $D(X)=D\left(\mathscr{A}_{X}\right) . \mathscr{A}$ is called locally free if the arrangements $\mathscr{A}_{X}$ are

Received by the editors November 19, 1991.

1991 Mathematics Subject Classification. Primary 05B35, 06A09, 14 F05. 
free for all $X \in L_{0}$. If $\mathscr{A}$ is free then it is locally free (see [T2]). The modules $D(X)$ with the natural inclusions form a sheaf $\mathscr{D}$ on $L_{0}$ provided with the order topology. The part of $L_{0}$ for $\mathscr{A}_{X}$ is played by the poset $L_{X}=\{Y \in L \mid Y>$ $X\}$, and we keep the symbol $\mathscr{D}$ for the restriction of the sheaf $\mathscr{D}$ to this poset. It was proved in [Y2] that $\mathscr{A}$ is free if and only if $H^{i}\left(L_{X}, \mathscr{D}\right)=0$ for every $X \in L$ and every $i, 0<i<\operatorname{codim} X-1$. Since the sheaf $\mathscr{D}$ is graded, its cohomology modules are graded, too, so the above condition amounts to the vanishing of their homogeneous components $H_{d}^{i}\left(L_{X}, \mathscr{D}\right)$ for all nonnegative degrees $d$.

One of the goals of this paper is to prove that for a locally free arrangement these linear spaces vanish if $d \geq d(L)$ where the bound $d(L)$ depends only on $L$. This result also implies that the Hilbert coefficients of $D(\mathscr{A})$ are combinatorial invariants if $\mathscr{A}$ is locally free. For the case $l=3$ this was proved in [RT, Corollary 3.4.3]. A similar technique gives an upper bound depending only on $L$ for the structure constants of a locally free arrangement.

Another goal of this paper is to prove that the set of all free arrangements is Zariski open in the set of all arrangements with a given intersection lattice. ${ }^{1}$ This result suggests the approach to Terao's conjecture by proving that the set of free arrangements is also Zariski closed, but the author does not see how to do it at the present time.

Some of the results of the paper were formulated in conversations with Terao during the author's visit to Madison. The author is grateful to Orlik and Terao for inviting him to Madison and for stimulating discussions.

\section{Notation}

Here we recall some definitions and set up the notation for the rest of the paper. Throughout $\mathscr{A}$ will be an $l$-arrangement of $n$ hyperplanes in a linear space $V$ (of dimension $l$ ) over an arbitrary field $K$, and for every $H \in \mathscr{A}$, $\alpha_{H}$ will be a fixed linear functional such that $\operatorname{ker} \alpha_{H}=H$. $\mathscr{A}$ will also denote the set of these functionals.

The graded ring $S$, the graded module $D=D(\mathscr{A})$, and the posets $L$ and $L_{0}$ were defined in the introduction. We denote by $M$ the irrelevant ideal of $S$, i.e., $M=\sum_{d>0} S_{d}$. Every $X \in L$ defines the subarrangement $\mathscr{A}_{X}$ of $\mathscr{A}$, which consists of the hyperplanes containing $X$. We put $D(X)=D\left(\mathscr{A}_{X}\right)$. The part of $L_{0}$ for $\mathscr{A}_{X}$ is played by the poset $L_{X}=\left\{Y \in L_{0} \mid Y>X\right\}$. Clearly $L_{U}=L_{0}$ where $U$ is the minimal element of $L$. The poset $L_{0}$ is graded by dimension. We put $L(q)=\left\{X \in L_{0} \mid \operatorname{dim} X=q\right\} \quad(q \geq 1)$. We will also use the poset $\bar{L}_{X}=\{Y \in L \mid Y \geq X\}$.

If $Q$ is an arbitrary finite poset then we view it as a topological space with the topology consisting of all increasing subsets of $Q$, i.e., subsets $R$ such that if $X \in R$ and $Y \geq X$ then $Y \in R$. The category of sheaves (of $S$-modules or linear spaces) on this topological space is isomorphic to the category of covariant functors on $Q$. In particular, a sheaf $\mathscr{F}$ on $Q$ is defined by the stalks $\mathscr{F}(X)$, $X \in Q$, and by the structure morphisms $\rho_{Y, X}: \mathscr{F}(X) \rightarrow \mathscr{F}(Y), X, Y \in Q$, $X<Y$, such that $\rho_{Y, Z} \rho_{X, Y}=\rho_{X, Z}$ for every $X<Y<Z$. A sheaf $\mathscr{F}$ on $Q$ induces a sheaf on any subset of $Q$, and we will keep the symbol $\mathscr{F}$ for this

\footnotetext{
${ }^{1}$ Discussing this result with my colleagues working in arrangement theory, I found out that while it seemed intuitively plausible to a few of them, it defied the intuition of the majority of them.
} 
sheaf. All information we need about the cohomology of sheaves can be found in $[\mathrm{G}]$.

An example of a sheaf important for arrangement theory is given by the sheaf $\mathscr{D}$ on $L_{0}$ defined by the stalks $\mathscr{D}(X)=D(X)$ and by the natural embeddings $D(X) \subset D(Y)$ for all $X<Y$ from $L_{0}$. For every $X \in L$ with $\operatorname{dim} X<l-1$ we have $H^{0}\left(L_{X}, \mathscr{D}\right) \simeq D(X)$. It is crucial for this paper that all stalks of $\mathscr{D}$ are graded $S$-modules and that the structure homomorphisms have degree 0 ; therefore, every cohomology module $H^{i}\left(L_{X}, \mathscr{D}\right)$ is graded too. We will denote the homogeneous component of degree $d$ of this module by $H_{d}^{i}\left(L_{X}, \mathscr{D}\right)$. For every graded $S$-module $N=\bigoplus_{d \geq 0} N_{d}$ we will denote by $P(N ; x)$ the Hilbert series of $N$, i.e., $P(N ; x)=\sum_{d \geq 0} \operatorname{dim} N_{d} x^{d}$. For sufficiently large $d$ the number $\operatorname{dim} N_{d}$ is the value at $d$ of a polynomial whose degree equals the Krull dimension of $N$ minus 1 and whose coefficients are called the Hilbert coefficients of $N$. In particular, the module $D(\mathscr{A})$ has the Krull dimension $l$ and the Hilbert coefficients of this module will be denoted by $h_{0}, h_{1}, \ldots, h_{l-1}$. If $\mathscr{A}$ is free then $P(D(\mathscr{A}) ; x)$ is defined by $L$ (see [T2]).

\section{A PROPERTY OF $S$}

In this section we prove a property of $S$ which will be used in the following section.

Proposition 1.1. Let $\left\{B_{1}, \ldots, B_{k}\right\}$ be a nonempty collection of finite nonempty subsets of $V^{*}=S_{1}$ satisfying the following condition:

for every choice of $\alpha_{i} \in B_{i} \quad(i=1, \ldots, k)$ these elements linearly generate $V^{*}$.

Then $\sum_{i=1}^{k} S \pi_{i} \supset M^{m}$ where $\pi_{i}=\prod_{\alpha \in B_{i}} \alpha$ and $m=\sum_{i=1}^{k}\left|B_{i}\right|$.

Proof. Fixing an arbitrary basis $\left(x_{1}, \ldots, x_{l}\right)$ of $V^{*}$ we can identify $S$ with $K\left[x_{1}, \ldots, x_{l}\right]$. Also without loss of generality we can assume that $\bigcup_{i=1}^{k} B_{i}$ does not contain 0 . First we use induction on $l$. If $l=1$ then every $\pi_{i}$ is a monomial in $x_{1}$ and the result is obvious. Suppose $l>1$. Now we apply induction on $m$. The minimal value of $m$ is $k$, and $m=k$ means that $\left|B_{i}\right|=1$ for every $i$. More generally let us suppose that $\left|B_{j}\right|=1$ for some $j \in\{1, \ldots, k\}$. Then we can assume that $B_{j}=\left\{x_{1}\right\}$. Factoring by the ideal $S x_{1}$, we obtain subsets $\bar{B}_{i}(i \in\{1, \ldots, k\} \backslash\{j\})$ of $\bar{S}=F\left[x_{2}, \ldots, x_{l}\right]$ that satisfy the condition $(*)$. Due to the inductive hypothesis (induction on $l$ ) the ideal of $\bar{S}$ generated by $\bar{\pi}_{i}$ contains $\bar{M}^{\bar{m}}$ where $\bar{M}$ is the irrelevant ideal of $\bar{S}, \bar{\pi}_{i}=\prod_{\alpha \in \bar{B}_{i}} \alpha$, and $\bar{m}=\sum_{i}\left|\bar{B}_{i}\right|$. Taking the preimages of these ideals in $S$ and using that $\pi_{j}=x_{1}$, we obtain the result.

Now suppose that $\left|B_{i}\right| \geq 2$ for each $i$, choose $\alpha_{i} \in B_{i} \quad(i=1, \ldots, k)$, and put $B_{i}^{\prime}=B_{i} \backslash\left\{\alpha_{i}\right\}$. For each $i$ the collection $\left\{C_{1}, \ldots, C_{k}\right\}$, where $C_{j}=B_{j}$ if $j \neq i$ and $C_{i}=B_{i}^{\prime}$, satisfies $(*)$. Thus by the inductive hypothesis (induction on $m$ ) the ideal $\sum_{j=1}^{k} S \pi_{j}$ of $S$ contains $\alpha_{i} M^{m-1}$ for every $i=1, \ldots, k$. Using property $(*)$ we have $\sum_{j=1}^{k} S \pi_{j} \supset M^{m}$, which completes the proof.

The following application of Proposition 1.1 is especially important for us. For each $X \in L(1)$ we put $B_{X}=\mathscr{A} \backslash \mathscr{A}_{X}$ and $\pi_{X}=\prod_{\alpha \in B_{X}} \alpha$. 
Lemma 1.2. If $\mathscr{A}$ is essential then the collection $\left\{B_{X} \mid X \in L(1)\right\}$ satisfies the condition $(*)$ of Proposition 1.1.

Proof. Since $\mathscr{A}$ is essential, all the sets $B_{X}$ are nonempty. Choose $\alpha_{X} \in B_{X}$ for each $X \in L(1)$ and suppose they do not linearly generate $V^{*}$. This means that the intersection of the respective hyperplanes is not 0 , whence there exists $Y \in L(1)$ such that $\alpha_{X} \in \mathscr{A}_{Y}$ for every $X \in L(1)$. Taking $X=Y$ we obtain a contradiction.

Corollary 1.3. The ideal $\sum_{X \in L(1)} S \pi_{X}$ of $S$ contains $M^{m(L)}$ where $m(L)=$ $\sum_{X \in L(1)}\left(n-\left|\mathscr{A}_{X}\right|\right)$.

For the rest of the paper we put $m=m(L)=\sum_{X \in L(1)}\left(n-\left|\mathscr{A}_{x}\right|\right)$.

\section{AN UPPER BOUND OF THE DEGREE OF NONZERO COHOMOLOGY}

In this section we assume that the arrangement $\mathscr{A}$ is essential and locally free and prove that there exists a number $d(L)$ depending only on $L$ such that $\widetilde{H}_{d}^{i}\left(L_{0}, \mathscr{D}\right)=0$ for every $d>d(L)$ and every $i$.

Fix an arbitrary basis $\left(x_{1}, \ldots, x_{l}\right)$ of $V^{*}$ and put $y_{i}=x_{i}^{m}$ where $i=$ $1, \ldots, l$ and $m=m(L)$ was defined in the previous section.

The properties of the elements $y_{i}$ which will be used are as follows.

(i) For every $i$ we have $y_{i} \in \sum_{X \in L(1)} S \pi_{X}$.

This follows immediately from Corollary 1.3 .

(ii) The system $\left(y_{1}, \ldots, y_{l}\right)$ is regular on $S$ (and thus on any free $S$ module).

Indeed the system $\left(x_{1}, \ldots, x_{l}\right)$ is a basis of $V^{*}$ and, thus, is regular. Due to [M, Theorem 16.1] the system $\left(y_{1}, \ldots, y_{l}\right)$ is regular.

Now for each $q, 0 \leq q \leq l$, we define the sheaf $\mathscr{D}^{q}$ on $L_{0}$, putting $\mathscr{D}^{0}=\mathscr{D}$ and $\mathscr{D}^{q}=\mathscr{D} / \sum_{i=1}^{q} y_{i} \mathscr{D}$ for $q>0$. By property (ii) $y_{q+1}$ is not a zero divisor on $\mathscr{D}^{q}(X)$ for every $q<l$ and $X \in L_{0}$. Thus we have the exact sequence of sheaves

$$
0 \rightarrow \mathscr{D}^{q} \stackrel{\phi_{q}}{\longrightarrow} \mathscr{D}^{q} \stackrel{\rho_{q}}{\longrightarrow} \mathscr{D}^{q+1} \rightarrow 0
$$

where the sheaf morphism $\phi_{q}$ is generated by multiplication by $y_{q+1}$ and $\rho_{q}$ is the natural projection. Both sheaf morphisms are homogeneous and $\rho_{q}$ preserves the degree while $\phi_{q}$ increases it by $m$.

Our nearest goal is to prove that for every $i>0$ the endomorphism $\phi_{q}^{*}$ of the module $H^{i}\left(L_{0}, \mathscr{D}^{q}\right)$ is 0 . First we fix $X \in L(1)$ and consider the morphism $\psi_{q}(X)$ of the sheaf $\mathscr{D}^{q}$ to itself generated by multiplication by $\pi_{X}$. The idea is to represent $\psi_{q}(X)$ as the composition of sheaf morphisms passing through an acyclic sheaf.

For that define the subsheaf $\mathscr{E}$ of $\mathscr{D}$ (for fixed $X \in L(1)$ ) putting $\mathscr{E}(Y)=$ $D\left(\mathscr{A}_{Y} \cup B_{X}\right) \quad\left(Y \in L_{0}\right)$. Then put $\mathscr{E}^{q}=\sum_{i=1}^{q} y_{i} \mathscr{E}$ and $\mathscr{F} q=\mathscr{E} / \mathscr{E} q$.

Lemma 2.1. The sheaf $\mathscr{F}^{q}$ is acyclic.

Proof. It follows directly from the definition that $\mathscr{F} q(Y)=\mathscr{F} q(Y \vee X)$ for every $Y \in L_{0}$. Thus we can apply [Y1, Lemma 1.2] to the pair of posets $\bar{L}_{X} \subset L_{0}$, the monotone map $Y \mapsto Y \vee X \quad\left(Y \in L_{0}\right)$, and the sheaf $\mathscr{F}^{q}$. According to this lemma $H^{i}\left(L_{0}, \mathscr{F} q\right) \simeq H^{i}\left(\bar{L}_{X}, \mathscr{F} q\right)$. Besides the sheaf, $\mathscr{F} q$ is acyclic on $\bar{L}_{X}$ due to [Y1, Lemma 1.1]. The result follows immediately. 
Lemma 2.2. There exist morphisms of sheaves $\xi_{1}: \mathscr{D}^{q} \rightarrow \mathscr{F}^{q}$ and $\xi_{2}: \mathscr{F}^{q} \rightarrow \mathscr{D}^{q}$ such that $\xi_{2} \xi_{1}=\psi_{q}(X)$.

Proof. For each $Y \in L_{0}$ we have $\pi_{X} D(Y) \subset D\left(\mathscr{A}_{Y} \cup B_{X}\right) \subset D(Y)$ and thus $\pi_{X} \sum_{i=1}^{q} y_{i} D(Y) \subset \sum_{i=1}^{q} y_{i} D\left(\mathscr{A}_{Y} \cup B_{X}\right) \subset \sum_{i=1}^{q} y_{i} D(Y)$. As $\xi_{1}$ and $\xi_{2}$ one can take the sheaf morphisms formed by these embeddings.

Proposition 2.3. The endomorphism $\psi_{q}^{*}(X)$ of $H^{i}\left(L_{0}, \mathscr{D} q\right)$ is 0 for every $i>0$. Proof. Follows immediately from Lemmas 2.1 and 2.2.

Corollary 2.4. The endomorphism $\phi_{q}^{*}$ of $H^{i}\left(L_{0}, \mathscr{D}^{q}\right)$ is 0 for every $i>0$.

Proof. Recall that $\phi_{q}$ is multiplication by $y_{q+1}$. According to property (i) $y_{q+1}=\sum_{i=1}^{k} f_{i} \pi_{X_{i}}$ for some $f_{i} \in S$ and $X_{i} \in L(1) \quad(i=1, \ldots, k)$. Thus $\phi_{k}^{*}$ is a linear combination over $S$ of the endomorphisms $\psi_{q}^{*}\left(X_{i}\right)$, which implies the result.

Corollary 2.5. For every $i>0$, every $d$, and $q=0,1, \ldots, l-1$ we have the exact sequences

$$
0 \rightarrow H^{i}\left(L_{0}, \mathscr{D}^{q}\right) \stackrel{\rho_{q}^{*}}{\longrightarrow} H^{i}\left(L_{0}, \mathscr{D}^{q+1}\right) \stackrel{\delta_{q}}{\longrightarrow} H^{i+1}\left(L_{0}, \mathscr{D}^{q}\right) \longrightarrow 0
$$

and

$$
0 \rightarrow H_{d}^{i}\left(L_{0}, \mathscr{D}^{q}\right) \rightarrow H_{d}^{i}\left(L_{0}, \mathscr{D}^{q+1}\right) \rightarrow H_{d-m}^{i+1}\left(L_{0}, \mathscr{D}^{q}\right) \rightarrow 0
$$

Proof. Applying Corollary 2.4 to the exact cohomology sequence induced by the short exact sequence (2.1) we obtain the sequences (2.2). The existence of exact sequences (2.3) follows from that due to the homogeneity of the homomorphisms $\rho_{q}$ and $\delta_{q}$.

Theorem 2.6. If $d>m(L) l+n-l$ and $i>0$ then $H_{d}^{i}\left(L_{0}, \mathscr{D}\right)=0$.

Proof. We prove the statement for every sheaf $\mathscr{D}^{q}$ by induction on $l-q$. First consider the case $q=l$. Note that the ideal $\sum_{i=1}^{l} S y_{i}$ of $S$ contains $M^{r}$ for every $r \geq m l$, whence every stalk $\mathscr{D}^{l}(X)$ of $\mathscr{D}^{l}$ is a module over the graded ring $S / M^{m l}$. As a system of generators of $\mathscr{D}^{l}(X)$, we can take the image of a system of homogeneous generators of $\mathscr{D}(X)$ whose degree are the exponents of $\mathscr{A}$ (see [T1]). Then there are $l$ of these degrees, exactly $\operatorname{dim} X$ of them are 0 , and their sum is $\left|\mathscr{A}_{X}\right|$. This implies that the degrees are bounded by $\left|\mathscr{A}_{X}\right|-l+\operatorname{dim} X+1$. Using also that $\left|\mathscr{A}_{X}\right|+\operatorname{dim} X \leq n$ for every $X \in L$ we see that $\mathscr{D}^{l}(X)_{d}=0$ for every $d>m l+n-l$, which implies the statement for $q=l$.

Now suppose $q<l$. Apply the exact sequence (2.3) for $i>0$ and $d>$ $m l+n-l$. By the inductive hypothesis, $H_{d}^{i}\left(L_{0}, \mathscr{D}^{q+1}\right)=0$, which implies that $H_{d}^{i}\left(L_{0}, \mathscr{D}^{q}\right)=0$. For $q=0$ we get the statement of the theorem.

Remark 2.7. Using the third term of the exact sequence (2.3) one can find sharper bounds on the degree of nonzero cohomology modules. In fact one can show by induction on $l-q$ that $H_{d}^{i}\left(L_{0}, \mathscr{D}^{q}\right)=0$ for every $d>$ $m(L)(l-i+1)+n-l, q \geq 0$, and $i>0$.

Informally, Theorem 2.6 asserts that there is a finite set of integer obstructions $\operatorname{dim} H_{d}^{i}\left(L_{0}, \mathscr{D}\right) \quad(i=1, \ldots, l-2, d=0,1, \ldots, d(L))$ to the freeness of a locally free arrangement with the lattice $L$. 
A formal corollary of the theorem is as follows.

Corollary 2.8. For a locally free arrangement $\mathscr{A}$ the Hilbert coefficients $h_{0}$, $h_{1}, \ldots, h_{l-1}$ of $\mathscr{D}(\mathscr{A})$ are defined by the lattice of intersections of $\mathscr{A}$.

Proof. Applying [Y1, Proposition 1.5] to $\mathscr{D}$ and using that $H^{0}\left(L_{0}, \mathscr{D}\right)$ $\simeq D$ we obtain

$$
P(D ; x)=-\sum_{i>0}(-1)^{i} P\left(H^{i}\left(L_{0}, \mathscr{D}\right) ; x\right)-\sum_{X \in L_{0}} \mu(0, X) P(D(X) ; x)
$$

where $\mu$ is the Möbius function of $L$. For $d>d(L)$ the coefficient of $x^{d}$ in the first sum in the right-hand side of $(2.4)$ is 0 due to Theorem 2.6. The same coefficient in the second sum depends only on $L$ since $\mathscr{A}$ is locally free. The result follows.

For the case $l=3$ Corollary 2.8 follows also from [RT, Corollary 3.4.3].

As still another use of the exact sequence (2.1) we prove that for a locally free arrangement with the intersection lattice $L$ the structure constants of this arrangement are bounded by a number depending only on $L$.

Proposition 2.9. All the structure constants of $\mathscr{A}$ are not greater than $p(L)=$ $p=m(L)(l+1)+n-l+1$.

Proof. Recall that the structure constants of $\mathscr{A}$ are the degrees of any minimal system of homogeneous generators of the $S$-module $D(\mathscr{A})=H^{0}\left(L_{0}, \mathscr{D}\right)$. We extend the statement to all modules $H^{0}\left(L_{0}, \mathscr{D}^{q}\right)(q=0,1, \ldots, l)$ and prove it by induction on $l-q$. If $q=l$ and $d>p>m l+n-l$ then it was shown in the first part of the proof of Theorem 2.6 that $\mathscr{D}_{d}^{l}=0$, whence the result is obvious. Assume that $q<l$ and $d \geq p$. The exact sequence (2.1) and Corollary 2.4 imply that the following sequence is exact:

$$
\begin{aligned}
0 \rightarrow H_{d-m}^{0}\left(L_{0}, \mathscr{D}^{q}\right) \stackrel{\phi_{a}^{*}}{\longrightarrow} H_{d}^{0}\left(L_{0}, \mathscr{D}^{q}\right) & \stackrel{\rho_{a}^{*}}{\rightarrow} H_{d}^{0}\left(L_{0}, \mathscr{D}^{q+1}\right) \\
& \rightarrow H_{d-m}^{1}\left(L_{0}, \mathscr{D}^{q}\right) \rightarrow 0 .
\end{aligned}
$$

Since $d-m>m l+n-l$ Theorem 2.6 implies that $H_{d-m}^{1}\left(L_{0}, \mathscr{D}^{q}\right)=0$, i.e., (2.5) becomes the short exact sequence

$$
0 \rightarrow H_{d-m}^{0}\left(L_{0}, \mathscr{D}^{q}\right) \stackrel{\phi_{q}^{*}}{\longrightarrow} H_{d}^{0}\left(L_{0}, \mathscr{D}^{q}\right) \stackrel{\rho_{q}^{*}}{\longrightarrow} H_{d}^{0}\left(L_{0}, \mathscr{D}^{q+1}\right) \longrightarrow 0 .
$$

By the inductive hypothesis we can fix homogeneous generators $t_{1}, \ldots, t_{k}$ of $H^{0}\left(L_{0}, \mathscr{D}^{q+1}\right)$ subject to the condition $\operatorname{deg}\left(t_{i}\right) \leq p$. Let $u_{1}, \ldots, u_{r}$ be the set of all the elements of $H_{p}^{0}\left(L_{0}, \mathscr{D}^{q+1}\right)$ which are the results of the action by monomials from $S$ on the elements $t_{i}$. Since $\rho_{q}^{*}$ is surjective for $d=p$, we can choose some preimages $v_{1}, \ldots, v_{r} \in H_{p}^{0}\left(L_{0}, \mathscr{D}^{q}\right)$ of $u_{1}, \ldots, u_{r}$. Then for every $h \in H_{d}^{0}\left(L_{0}, \mathscr{D}^{q}\right)$ there exist $s_{1}, \ldots, s_{r} \in S$ such that $h^{\prime}=h-\sum_{i=1}^{r} s_{i} v_{i} \in$ $\operatorname{Im} \phi_{q}^{*}$, i.e., $h^{\prime} \in y_{q+1} H_{d-m}^{0}\left(L_{0}, \mathscr{D}^{q}\right)$. In particular, this implies that $h$ is an $S$-linear combination of the elements $v_{1}, \ldots, v_{r}$ (of degree $p \leq d$ ) and of some elements of degrees lower than $d$, which completes the proof.

Remark 2.10. It is quite probable that the estimate for the structure constants given in Proposition 2.9 is far from being sharp. I do not know of any example of a locally free arrangement with structure constants larger than $n-l$. 


\section{Free ARRANGEMENTS With A GIVEN INTERSECTION LATTICE}

In this section we fix a geometric lattice $L$ and study the set of all essential arrangements whose intersection lattice is isomorphic to $L$. More exactly we consider the quasi-affine variety $\mathscr{V}$ of all $n \times l$ matrices $\left(a_{i j}\right)$ of rank $l$ over $F$ with certain minors 0 and others nonzero so that the lattice of intersections of the hyperplanes defined by the rows is isomorphic to $L$. We view $\mathscr{V}$ as a topological space with the Zariski topology and call its elements arrangements (slightly abusing this term).

Denote by $\mathscr{W}$ the subset of $\mathscr{V}$ of all locally free arrangements (as it will be proved later $\mathscr{W}$ is open in $\mathscr{V}$ ). Fix a nonnegative number $d$. To every arrangement from $\mathscr{W}$ we assign the homogeneous component of degree $d$ of the cochain complex (1) from [Y1] whose homology is $\widetilde{H}_{d}^{*}\left(L_{0}, \mathscr{D}\right)$. Since the arrangement is locally free, $\operatorname{dim} D(X)_{d}$ depends only on $L$ for every $X \in L_{0}$. Thus the above assignment defines the map $f_{d}=f$ from $\mathscr{W}$ to the set $\mathscr{C}=$ $\mathscr{C}(d)$ of all cochain complexes

$$
F^{k_{0}} \rightarrow F^{k_{1}} \rightarrow \cdots \rightarrow F^{k_{l-1}}
$$

where $k_{i}=\sum_{\operatorname{dim} X=i+1}|\mu(0, X)| \operatorname{dim} D(X)_{d} \quad(i=0,1, \ldots, l-1)$. The set $\mathscr{C}$ can be identified with the set of all $(l-1)$-tuples of matrices $\left(\partial_{1}, \ldots, \partial_{l-1}\right)$ $\left(\partial_{i}\right.$ being a $\left(k_{i-1} \times k_{i}\right)$-matrix over $\left.K\right)$ subject to the conditions $\partial_{i} \partial_{i+1}=0$ $(i=1, \ldots, l-2)$. This identification provides $\mathscr{C}$ with the structure of an affine algebraic variety. By $\mathscr{C}_{0}=\mathscr{C}_{0}(d)$ we denote the subset of $\mathscr{C}(d)$ of all complexes that are exact. Providing $\mathscr{C}$ with the Zariski topology we make the following observation.

Lemma 3.1. The set $\mathscr{C}_{0}$ is open in $\mathscr{C}$.

Proof. It suffices to prove that the set $\mathscr{C}^{j}$ of all complexes from $\mathscr{C}$, which are exact in the term $F^{k_{j}}$, is open in $\mathscr{C}$ for every $j=1, \ldots, l-2$. Thus fix $j$ and consider the corresponding part of a complex

$$
\cdots \rightarrow F^{k_{j-1}} \stackrel{\partial^{\prime}}{\longrightarrow} F^{k_{j}} \stackrel{\partial^{\prime \prime}}{\longrightarrow} F^{k_{j+1}} \rightarrow \cdots .
$$

We have $\mathscr{C} \backslash \mathscr{C}^{j}=\bigcup_{i=0}^{k_{j}} \mathscr{N}_{i}$ where $\mathscr{N}_{i}$ is the set of all complexes satisfying the conditions $\operatorname{dim} \operatorname{Im} \partial^{\prime}<i$ and $\operatorname{dim} \operatorname{Ker} \partial^{\prime \prime} \geq i$ (some of the sets may be empty). Each $\mathscr{N}_{i}$ is an affine subvariety of $\mathscr{C}$ whose ideal in the coordinate ring of $\mathscr{C}$ is generated by all $i \times i$ minors of $\partial^{\prime}$ and $\left(k_{j}-i\right) \times\left(k_{j}-i\right)$ minors of $\partial^{\prime \prime}$. Thus $\mathscr{C}^{j}$ is open in $\mathscr{C}$, which completes the proof.

Lemma 3.2. The map $f$ is rational.

Proof. For each $X \in L_{0}$ consider the linear space $D(X)_{d}$ as a subspace of $S_{d}^{l}$ and fix the standard monomial basis of $S_{d}^{l}$. The subspace $D(X)_{d}$ is defined with respect to the basis by linear equations whose coefficients are rational functions of the entries $a_{i j}$. Thus one can choose a basis of $D(X)_{d}$, whose elements are $l$-tuples of polynomials of degree $d$ with rational functions of $a_{i j}$ as the coefficients. Then for every $X, Y \in L_{0}$ such that $X \subset Y$ the matrix of the embedding $D(X)_{d} \subset D(Y)_{d}$ has rational functions of $a_{i j}$ as the entries, whence the same is true about the matrices of the differentials of the respective cochain complex. 
Theorem 3.3. The set of all free arrangements is open in $\mathscr{W}$.

Proof. According to [Y2, Theorem 1.1] free arrangements form the set $\bigcap_{d \geq 0} f_{d}^{-1}\left(\mathscr{C}_{0}(d)\right)$. Due to Theorem 2.6 we need to consider only a finite number of values of $d$ in this intersection. Thus the result follows immediately from Lemmas 3.1 and 3.2.

Corollary 3.4. The set of all free arrangements is open in $\mathscr{V}$.

Proof. Apply the induction on $l$. If $l=2$ all arrangements are free. Suppose $l>2$ and fix $X \in L_{0}$. The arrangement $\mathscr{A}_{X}$ is not essential, but factoring its elements by $X$ we can associate to it the essential $(l-\operatorname{dim} X)$-arrangement $\mathscr{A}_{X}^{\prime}$ in $V / X$. These two arrangements have the same intersection lattice $\bar{L}_{X}$, and $\mathscr{A}_{X}$ is free if and only if $\mathscr{A}_{X}^{\prime}$ is free (cf. [Y2]). Also for any choice of basis in $V / X$ the assignment $\mathscr{A} \mapsto \mathscr{A}_{X}^{\prime}$ is given by linear functions of the entries $\left(a_{i j}\right)$ of $\mathscr{A}$. Using the inductive hypothesis we see that the set of all arrangements $\mathscr{A}$ from $\mathscr{V}$ with $\mathscr{A}_{X}$ free form an open set. Thus the locally free arrangements form an open set in $\mathscr{V}$. Now the result follows from Theorem 3.3.

\section{REFERENCES}

[G] R. Godement, Topologie algébrique et théorie des faisceaux, Hermann, Paris, 1958.

[M] H. Matsumura, Commutative algebra, Benjamin, New York, 1970.

[RT] L. Rose and H. Terao, Hilbert polynomials and geometric lattices, Adv. Math. 84 (1990), 209-225.

[T1] H. Terao, Arrangements of hyperplanes and their freeness. I, J. Fac. Sci. Univ. Tokyo Sect. IA Math. 27 (1980), 293-312.

[T2] _ Generalized exponents of a free arrangement of hyperplanes and Shephard-ToddBrieskorn formula, Invent. Math. 63 (1981), 159-179.

[T3] _ Free arrangements of hyperplanes over an arbitrary field, Proc. Japan Acad. Ser. A 59 (1987), 301-304.

[Y1] S. Yuzvinsky, Cohomology of local sheaves on arrangement lattices, Proc. Amer. Math. Soc. 112 (1991), 1207-1217.

[Y2] _ The first two obstructions to the freeness of arrangements, Trans. Amer. Math. Soc. 335 (1993), 231-244.

Department of Mathematics, University of Oregon, Eugene, Oregon 97403

E-mail address: yuz@MATH.uoregon.edu 